\title{
Construction of Health Promotion Management System Based on Web
}

\author{
Weibing $\mathrm{WU}^{\mathrm{a}}$, Xiaodan $\mathrm{LIU}^{\mathrm{b}}$, Lijuan $\mathrm{YU}^{\mathrm{a}}$ \\ ${ }^{a}$ School of Sports Science, Shanghai University of Sport, Shanghai, China \\ ${ }^{b}$ The Second Clinic School, Nanjing University of Traditional Chinese Medicine, Nanjing, China
}

\begin{abstract}
Health management is a new service for healthy and sub-healthy people in modern medical institutions, which will help to instruct people how to effectively control and maintain their health. Through questionnaire, systematic analysis and software engineering, the structure and function of health promotion management system are analyzed and designed. Finally, the health promotion management system based on web is constructed. People become willing to take advantage of the system to manage and direct their own health instead of becoming victims of diseases.
\end{abstract}

Index Terms: Health promotion; management system; web; design; construction

(C) 2011 Published by MECS Publisher. Selection and/or peer review under responsibility of the Research Association of Modern Education and Computer Science.

\section{Introduction}

The number of lifestyle-related chronic diseases has been raising worldwide and medical expenses unbearable, which has placed heavy burdens on families and the society. How to transfer the focus from-diagnosing patients in big hospitals to managing people's health and diseases in communities is an important and pressing task to us. . Health management is a personalized health management service based on the personal health records. It is established on the mode of modern biomedicine and information management technology, to ensure individual's health from the aspects of society, psychology and biology, and help people to control their health condition. Its core contents are: collecting individual health information, building health files, assessing health condition, predicting health tendency, enacting and executing health plans. By implementing health management, people manage their own health and reduce medical expenses instead of becoming victims of diseases.

* Corresponding author.

E-mail address:wwb75@126.com 


\section{The idea of system design and research methods}

A. The idea of system design

Health management is a comprehensive process that monitors, analyzes and evaluates the health of individuals or groups and provides the health advice and guidance on health, and interferes the risk factors of health. The purpose of health management is to inspire the enthusiasm of individuals or groups, and to use the limited resource effectively for the best health effect [2].

The basic idea of the health promotion management system design is:

- Firstly, provide the clients a complete and detailed health profiles, recording the changes of health.

- Then, use the health information to analyze the health condition effectively, to predict and support related medical and health knowledge.

- Finally, maintain health, instruct and manage personal life and behavior style by executing the health promotion plans.

B. Research methods

1) Interview survey: Talk with experts of health management, sports medicine and information management by semi-structured interviewing. Analyze the exercise health promotion management system and then report.

2) Systemic analysis: Based on systemic viewpoint, this paper synthetically analyzes the influencing factors of health risk assessment. These influencing factors include disease history, life style, physical quality testing, medical examination and so on. Then, the model of health risk assessment is established and instructs health promotion plans [3].

3) Software engineering: The system design adopts the method of life cycle, and Microsoft SQL Server 2000 is chosen as the back-end database under Windows XP operating system.

\section{The demand analysis of the system}

Health management is a new concept for Chinese because it appeared in China no more than 10 years ago. Now in China there are mainly two kinds of institutions providing health management service: hospitals and health management companies [4]. These institutions can provide the good service to the customers who own high income, because this service is a high consumption. There are already some health management systems for the ordinary families, but these systems' function is not rich enough to satisfy some of the families' needs, such as heath risk assessment and the provision of the health-related knowledge. So we need a health management system which not only suit ordinary families, but also provide abundant health management functions [5] 


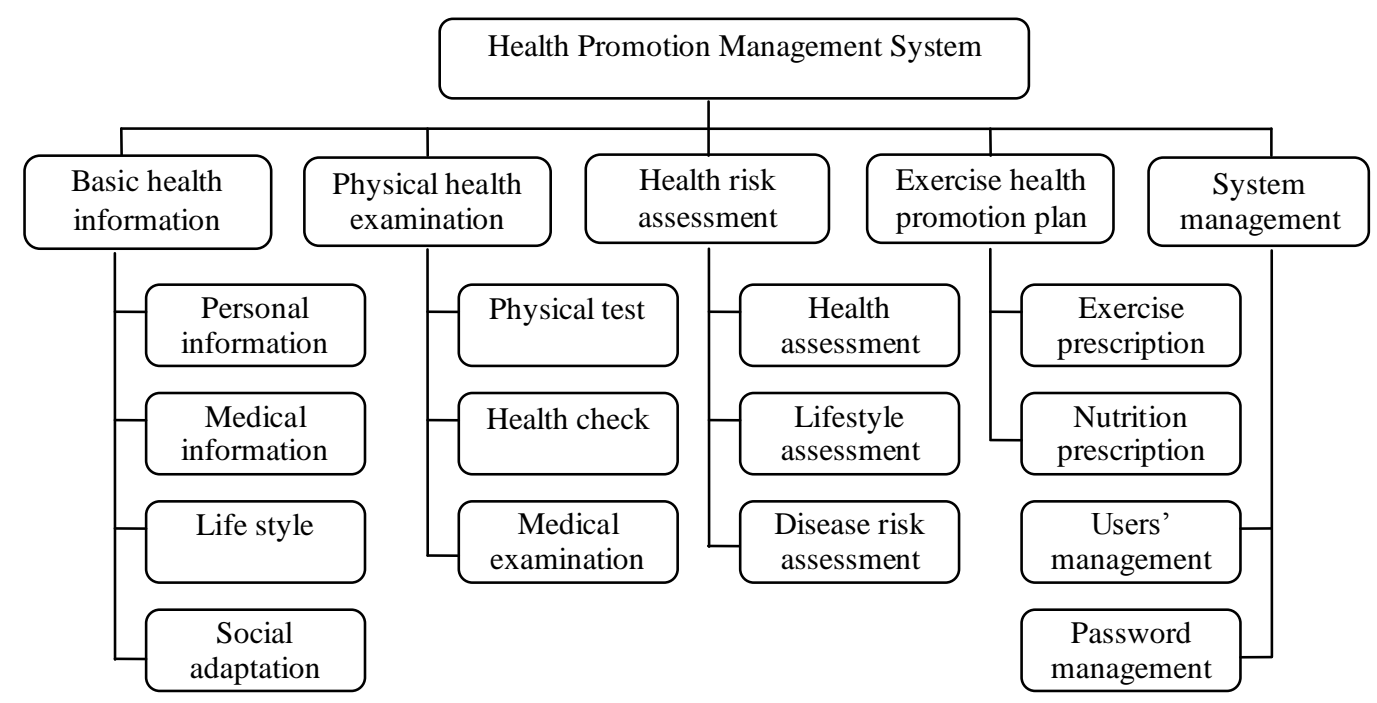

Fig.1 The structure of health promotion management system

\section{The general plan of system}

A. The structure of system

In accordance with the demand analysis of health promotion management system, the system is divided into 5 subsystems:

- basic health information management subsystem

- $\quad$ physical health examination management subsystem

- $\quad$ health risk assessment subsystem

- health promotion management subsystem

- $\quad$ system information management subsystem

As is shown in Fig. 1.

B. Main function of the system

This system serves as a monitor of individual health condition, and helps people to maintain health through providing exercise related methods, thus fulfilling the goal of promoting and managing peoples' health. The four main functions are as follows: health management, data acquisition, information analysis and health guidance [6]. 1) Management function

a) Parameters of management center such as name, icon, numbers of involved doctors, password and nutrients arrangement can be set in the system; software upgrade and data update are also available.

b) Through e-file management, doctors can get easy access to each client's basic information, and help to manage their record of health history, which includes record of clientsclients' disease treatment and effect evaluation, health instructions on exercise and diets, assessment of health risk factors each time and so on.

c) Health examination management makes personalized examining program and arranges appropriate regular examination, which are followed by result evaluation and health guidance. All of the above will be included in a comprehensive health managing procedure.

d) Data management is responsible for the database compressing, backup uploading, data query, data import and export, and statistical analysis.

2) Data acquisition function 
e) Physical examination data acquisition inputs results of lab and physical tests in routine format according to the requirement of the health managing system, and old units can be converted into new ones automatically. Results of other personalized tests related to clientsclients' health may be in non-fixed format.

f) Exercise information acquisition is conducted by an energy monitor which is linked to a computer, by collecting daily and previous exercise information recorded in the monitor. Doctors in distant hospitals can obtain the data through the Internet and make reasonable health recommendations [7].

g) Dietary information is entered in standard dietary diary format defined by the system. Non-standardized format is also permitted for different kinds of food and drink. The diary can be updated through the Internet as well.

3) Analysis function

h) Health examination analysis can draw a trend graph of all test results of all time and indicate the risk factors of possible diseases, based on the test results and health examination survey.

i) Exercise analysis examines the amount, intensity, duration and time of exercise through the historical exercise trend graph.

j) Through clients' dietary diary, nutrients analysis is able to assess the rationality of total amount of daily intake, proportion of macronutrient intake in total calorie and each meal's proportion in total calorie.

4) Guidance function

k) The individual health guidance plan provides guidelines of personal health risk factors in different periods through information of medical examinations and health investigations.

1) During clients' further consultation each time, the periodical diet guidance plan provides guidelines of next-stage diet, including intelligent food match and routine food match, in accordance with the information of exercise and dietary diary provided.

$\mathrm{m})$ The periodical exercise guidance plan provides guidelines of next-stage exercise, including guidance of total amount of exercise, guidance of effective amount of exercise, exercise intensity, exercise frequency, mode of exercise, exercise heart rate, exercise duration, also through information of exercise and dietary diary provided by clients during further consultation each time.

\section{Architecture of the system}

In architecture, $\mathrm{B} / \mathrm{S}$ is able to interact with server side and client side by the Internet. The client side includes users and browser and the server side includes web server, application server, data base and model base [8].

The running process of the architecture is as follows:

- The browser provides users a friendly interface, users send request to web server through the browser.

- Web server responds and exchanges information with the application server.

- The application server requests the data and model from data base and model base and provides to users in the form of web pages after background process.

The advantage of the architecture lies in meeting users' requirement of decision-making, and realizing fast delivering and sharing of decision resource sharing [9]. As is shown in Fig. 2.

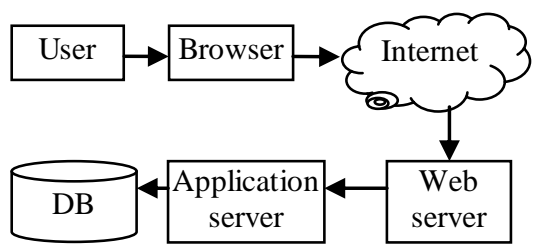

Fig. 2 The architecture of the system 


\section{The design of system}

The health promotion management system's solution is designed in accordance with the function demand of the health promotion management system and the business procedure analysis in the project, using such technologies as dynamic web page, database, data structure, network communication etc. It solves especially the key problems such as systematic system structure, generalization and modularity of system, generating checkup report documents automatically and system security design.

This system designs four-layer stored structure of checkup items after analyzing the characteristics of many check items of health promotion management center and realizes dynamic loading and organizing controls in the page using the control "Table" of ASP.NET, so it has great generalization. It divides the function of check-up and report into many little independent logical modules and then uses user control to realize the little module's function so that the upgrade and maintenance is very easy [10].

Many flexible universal word templates are designed in order to ensure the uniformization and criterion of physical checkup report. This system uses the method which generates the word documents in server and then downloads to clients after comparing the advantages with the disadvantages of three methods of generating word documents in web [11]. To ensure the security of system, form authentication technique and authorization technique in ASP. NET is applied to avoid illegal access and unauthorized operation using the theory of rolebased access control.

\section{Conclusions}

The health promotion management system is constructed by the application of modern computer network technology and management science. The health promotion management system based on web can increase checkup efficiency, standardize checkup medical affairs, facilitate checkup crowd and provide credible elaborate checkup information for medical research, so it is important for improving medical prevent level and civil health level. Because the system is of complex construction, involving wide technology and higher standard for reliability, real time reaction, generality and security, the construction of the system is mainly discussed in this paper.

\section{Acknowledgments}

We acknowledge the initial guidance from Dr Peiliang LING and thank Dr Xianming MENG for technical assistance. This study is supported by Shanghai leading academic discipline project under grant (no.s30802) and the specific plan for local academy construction of Shanghai science and technology commission under grant (no.10490503500).

\section{References}

[1] Investigation of Anhui academy government, "Health crisis of civil servants in the middle," Decision, Vol.56, pp.46-48, 2010(In Chinese).

[2] W.B. Wu and L.J. Yu, "Study of decision support system for body function monitoring in training," Journal of shanghai university of sports, Vol.31, pp.55-59, 2007(In Chinese).

[3] Z.L. Wen and M. Wang, "Health quality is the foundation of civil servants harmonious development," Chinese administration management, Vol.132, pp.25-27, 2008(In Chinese).

[4] J.M. Bao, "The design and realization of physical checkup management system based on web," Dalian: Dalian university of technology, 2005(In Chinese).

[5] J.S. Chen and J.S. Huang, "Health management specialist," Beijing: Peking union medical college press, 2007(In Chinese). 
[6] A.P. Sun, "Health management practical technology," Beijing: Chinese medicine and technology press, 2009(In Chinese).

[7] G. Ma, D.B. Wang and Q. Hong, "Explore of management model of middle-aged population health," Modern preventive medicine, Vol.37, pp.58-63, 2010.

[8] L.H. Tang, C.D. Dian and L.C. Tang, "The current research status and rethink of sports health promotion," Journal of tianjing university of sports, Vol.25, pp.201-205, 2010(In Chinese).

[9] Z.L. Gu. "Analysis and application about accessing technology of SQL sever by ADO," Computer applications and software, Vol.21, pp.32-33, 2004.

[10] K.S. Bai. "Visual Basic programming," Beijing: Tsinghua university press, 2006(In Chinese).

[11] K.S. Wang and Y.T. Song. "Development of mine safety production information system base on SQL database," Journal of coal science and technology, Vol.35, pp.56-58, 2007(In Chinese). 